\title{
AMPLITUDE - PHASE DIFFRACTION GRATINGS BASED ON THIN LAYER OF INDIUM TIN OXIDE
}

\author{
V.D. Paranin ${ }^{1}$, S.V. Karpeev ${ }^{1,2}$ \\ ${ }^{1}$ Samara National Research University, Samara, Russia \\ ${ }^{2}$ Image Processing Systems Institute - Branch of the Federal Scientific Research Centre \\ "Crystallography and Photonics" of Russian Academy of Sciences, Samara, Russia
}

\begin{abstract}
It is offered to operate the transmittance of a diffraction mask by changing the interference properties of a thin optical layer. Based on the results of the offered approach the amplitude-phase diffraction grating with the period of 300 microns has been made from the $115 \mathrm{~nm}$ transparent indium tin oxide (ITO) film. Optical and dimensional parameters of the diffraction mask have been investigated by using the ellipsometry, interferometry and spectroscopy. Computer modeling of the diffraction scattering and its experimental investigation have been carried out.
\end{abstract}

Keywords: amplitude-phase diffraction grating, thin layer, indium tin oxide, ellipsometry, interferometry, spectroscopy

Citation: Paranin VD, Karpeev SV. Amplitude - phase diffraction gratings based on thin layer of indium tin oxide. CEUR Workshop Proceedings, 2016; 1638: 95-102. DOI: 10.18287/1613-0073-2016-1638-95-102

\section{Introduction}

Diffraction gratings find application in imaging spectral devices [1,2] and optoelectronic actuator position sensors [3,4]. Most of the gratings used are phase gratings manufactured either by cutting using a ruling machine $[1,2]$ or by lithographical methods [5]. Thermochemical chrome oxidation remains the most developed and common method of producing lithographic masks and diffraction optical elements (DOEs) both for binary [6,7] and gray-scale DOEs [8]. Comparatively recently research has been underway devoted to further development of the technology of the additive method of producing phase grating microrelief $[9,10]$. The technology proposed consists in thermal oxidation of a thin metal molybdenum layer $(15-70 \mathrm{~nm})$. It may be followed by substrate etching through the mask produced. Let us note that variations in the temperature and time of annealing directly affect the chemical composition and structure (i.e. the optical thickness and absorption) of the oxide film. Consequently, it becomes possible to produce all types of gratings: amplitude, phase and amplitude-phase ones using the method specified. Besides, gratings incorporating 
opaque metal masks can be used as reflective ones without spraying or depositing additional layers [11]. It is possible to tune the parameters of such gratings to a given wavelength, for instance, by additional annealing at the post-treatment stage.

Further improvement of the grating functionality is achieved by using multilayer diffraction masks based on alternating optical layers. This makes it possible to increase the difference in the transmittance of the diffraction grating step and trench for a prescribed introduced phase difference. The additive technology makes it possible to produce diffraction gratings on the basis of various chemical compounds, including multilayer implementation. In this case oxidation of several metal layers tens of nanometers thick is a lot easier to accomplish than etching of several heterogeneous oxides of metals and/or semiconductors of greater thickness.

The possibility of producing diffraction masks possessing some peculiar properties, for instance, intended for application in electrically-controlled diffraction gratings is a version of development of the additive method [12-16]. As a result, diffraction elements of optical radiation control using various functional effects: electro-optical, magneto-optical, thermo-optical etc. can be implemented. It certainly requires the replacement of conventional quartz substrates by crystal or ceramic materials with the necessary functional properties. Besides, it gives birth to new requirements to the pattern of the diffraction mask. For example, electro-optical effects of various orders can be used in the case of interdigital electrodes. Magneto-optic and thermo-optic effects are used in the case of closed-circuit electrodes.

The aim of this work was the production and experimental investigation of a diffraction grating on the basis of a conducting mask made of indium-tin oxide (ITO) and measurement of ITO-layers' optical and dimensional characteristics by methods of ellipsometry, interferometry and spectroscopy. Directional diagrams of an ITOgrating were modeled and experimentally analyzed on the basis of the optical characteristics measured.

\section{Technology of ITO-grating production}

K-8 glass with the dimensions $25 \times 25 \times 3 \mathrm{~mm}$ was used as the grating substrate. Indium- tin oxide In2O3-SnO2 of electronic grade deposited by reactive magnetron sputtering in vacuum served as the material of the diffraction mask.

The substrates were cleaned by rinsing in an alkaline solution, soaking in a chrome solution $\mathrm{H} 2 \mathrm{SO} 4: \mathrm{K} 2 \mathrm{CrO} 7$ for 5 minutes and rinsing in deionized water. The lift-off process was used to produce a diffraction grating [17]. A layer of FP-051K photoresist $0.4 \mu \mathrm{m}$ thick was spin-deposited in two stages: 20 seconds at the rate of $500 \mathrm{rpm}$, and then 45 seconds at the rate of $4500 \mathrm{rpm}$. The photoresist was air-dried for 2 minutes and then dried on a heater for 12 minutes at the temperature $95{ }^{\circ} \mathrm{C}$. Immediately after the inverse-photomasked exposure the photoresist was developed in UBP- 
1F developer solution for 1 minute, rinsed in deionized water and dried by pure dried air flow.

The next step was the formation of indium- tin oxide by reactive sputtering in oxygen with additional heating. In order to accomplish vacuum deposition of the indium-tin layer the substrates with the photoresist inverse mask were fixed on a heating substrate holder of the ETNA-100-MT vacuum unit. The temperature of heating was limited by possible photomask degradation. It was experimentally established that heating up to the temperature of $200{ }^{\circ} \mathrm{C}$ at the rate of $10^{\circ} \mathrm{C} / \mathrm{min}$ in the presence of an argon-oxygen mask does not cause any significant variation of the properties of the photoresist used. Therefore, the temperature of the substrate holder was maintained at the level of $200^{\circ} \mathrm{C}$ in the course of the whole process of indium-tin deposition.

The process of sputtering started with spraying of the upper layer of the target material, the electronic grade indium-tin alloy (target diameter 3") onto the closed shutter under the following conditions: atmosphere- argon with the chamber pressure 0.5 mtorr, the rate of supply through the magnetron- $9 \mathrm{~cm} 3 / \mathrm{sec}$; the magnetron power increased up to $200 \mathrm{~W}$ in the course of 30 seconds and then was maintained at this level for 5 minutes in direct current conditions. Let us note that the closed magnetron shutter and the supply of gas from the bottom of the magnetron determined higher argon working pressure in the magnetron as compared to that in the main chamber, which ensured stable operation of the magnetron.

After the target was cleaned the shutter was opened, the flow of argon through the magnetron increased up to $22 \mathrm{~cm} 3 / \mathrm{sec}$, with additional supply of oxygen to the substrate at a rate of consumption $9 \mathrm{~cm} 3 / \mathrm{sec}$ (the pressure in the chamber therewith rose up to $1.1 \mathrm{mtorr})$. The magnetron power decreased down to $110 \mathrm{~W}$ and its operation condition changed to the discontinuos conduction mode with a periodic inverted pulse to minimize the effect of anode loss. The film thickness was controlled with the help of a quartz thickness gage using a witness placed near the specimens. The rate of ITO oxide formation on the witness is somewhat different, consequently, the film density may also differ from that on the specimen film. Therefore, to control the process the rate of film growth was used (it amounted to $0.26 \mathrm{~nm} / \mathrm{sec}$ ), the constancy of which meant the process was stable.

A diffraction grating made of indium-tin oxide on a K-8 substrate was formed in the way described. The grating dimensions were controlled by an automatic WLI-DMR white-light interferometer and were as follows: the period $-300 \mu \mathrm{m}$, the groove width - $165 \mu \mathrm{m}$, the microrelief depth $-115 . .117 \mathrm{~nm}$ (fig.1).

The refraction index of the ITO-film was measured by the method of ellipsometry using a J.A.Woollam V-VASE automatic spectral ellipsometer. The wavelength was assigned to be equal to $632.8 \mathrm{~nm}$, the width of the radiation spectrum did not exceed $2.3 \mathrm{~nm}$. To reduce the impact of reflection from the back surface of the substrate it was mat-finished. The results of measuring the intensity of reflection of p- and spolarized radiation are shown in fig. 2 . The refraction and absorption indices deter- 
mined with the help of the ellipsometer software were as follows: $n=1.857 \pm 0.008$, $\mathrm{k}=0.000 \pm 0.009$ according to the data of s-polarization. The refraction indices presented are averaged due to the structural complexity and possible heterogeneity of the ITO-film over its thickness.

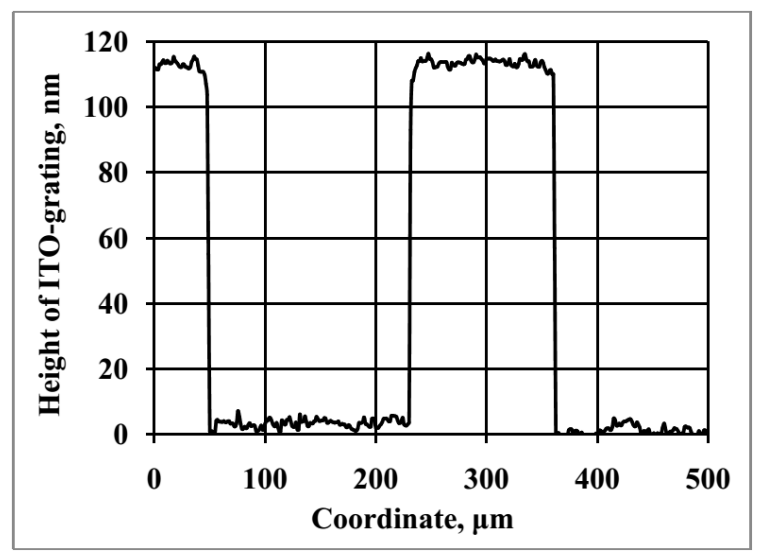

Fig. 1. ITO grating profile

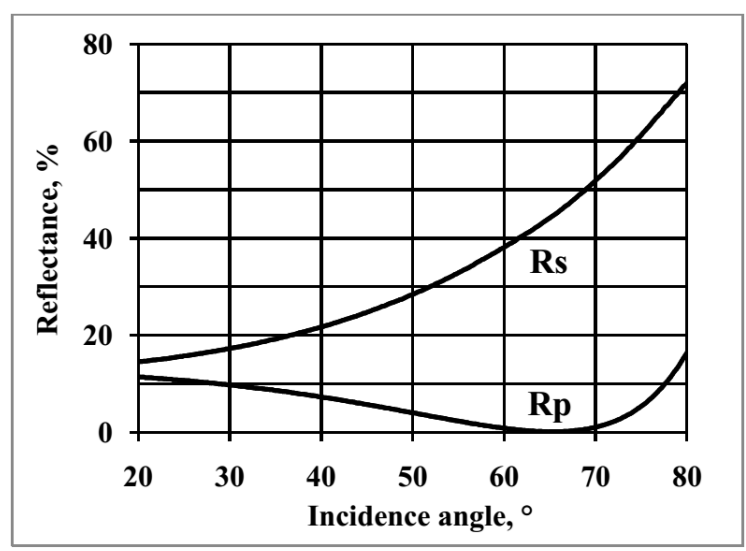

Fig. 2. Intensity of reflection of p-and the s-polarized light

Spectral transmittance of the substrate and the substrate with an ITO-layer in the range of 300-900 $\mathrm{nm}$ was investigated on a Shimadzu UV-2450 spectrophotometer. The measuring interval was $1 \mathrm{~nm}$, the spectral width of the slit $-1 \mathrm{~nm}$, with an average scanning speed. The results are presented in fig. 3 .

Maximum transmittance, practically equal to the substrate transmittance can be observed at the wavelength of $480 \mathrm{~nm}$. This maximum corresponds to the half-wave thickness of the ITO film. According to reference data the refractive index at the wavelength of $480 \mathrm{~nm}$ is $\mathrm{n}=1.996$. Thus, it is possible to determine the physical thick- 
ness of the film as $120 \mathrm{~nm}$, which is quite close to the results of measuring presented in fig. 1 .

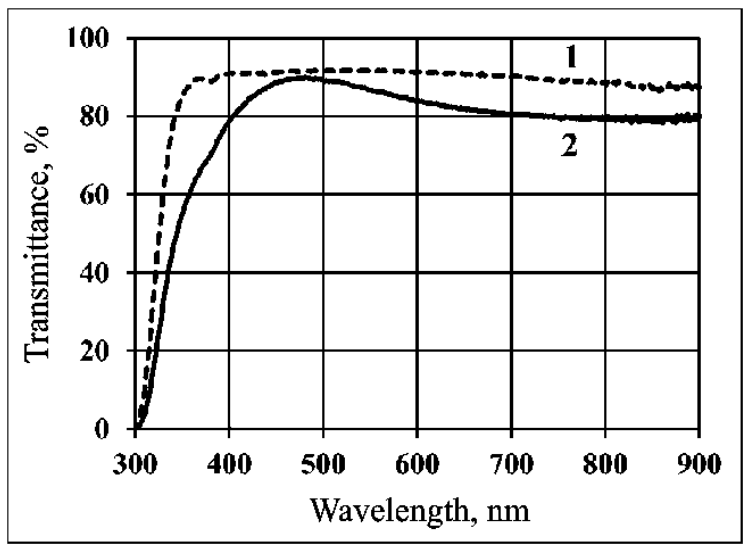

Fig. 3. Spectral transmittance of samples: 1 - a substrate, 2 - a substrate with an ITO film

\section{Analysis of ITO-grating characteristics}

Intensity distribution in the image plane was calculated on the basis of measuring the ITO-grating profile and refraction index. The Rayleigh-Sommerfeld diffraction integral for a one-dimensional structure was used in the calculation. The model grating comprised 30 slits with a period of $30 \mu \mathrm{m}$ and the slit width of $165 \mu \mathrm{m}$. The height of the ITO-profile was assigned equal to $115 \mathrm{~nm}$, the refraction index -1.857 . The intensity transmittance with account of the lower surface of the substrate in the area of ITO was $0.826 \mathrm{rad}$, in the region of the slit -0.190 . The phase change in the ITO area was 2.123 in the area of the slit $-1.142 \mathrm{rad}$. The results of calculating standardized intensity in the image plane $(0.4 \mathrm{~m}$ from the grating) are shown in fig. 4 .

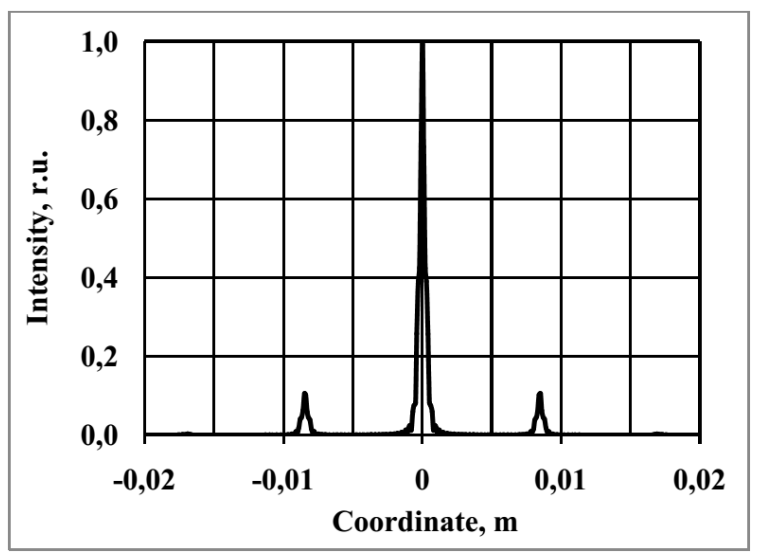

Fig. 4. Calculated distribution of intensity in the picture plane of an ITO grating 
The ITO-grating was investigated on an optical setup comprising a LGN-208A helium-neon laser, a spatial filter - beam expander, an optical-grade film polarizer, an experimental specimen, an imaging cylindrical lens, a photosensitive device. The intensity in the focal plane of the cylindrical lens was registered by a DCM310 CCDcamera with the $2048 \times 1536$ resolution. The illumination of the diffraction grating was close to uniform. Intensity distribution in the plane of the CCD-matrix is shown in fig.5.

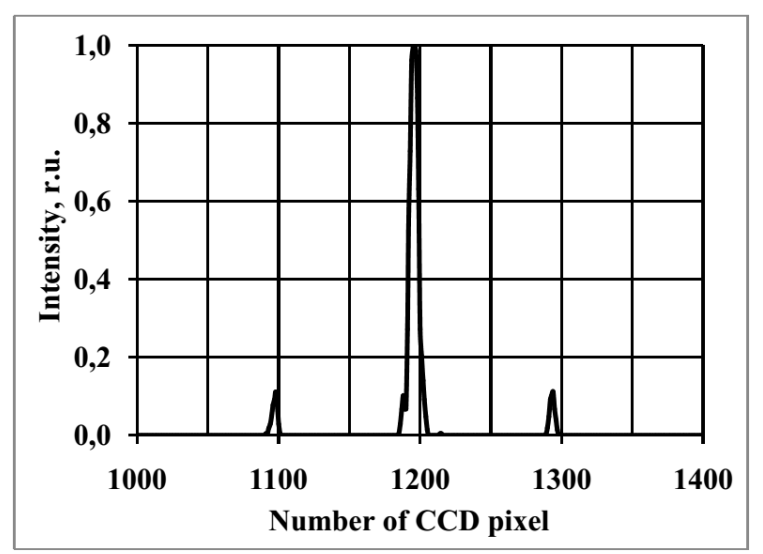

Fig. 5. Experimental distribution of intensity in the picture plane of an ITO grating

Comparison of fig.4 and fig.5 shows good agreement between the experimental and design characteristics of the grating. It follows from the data obtained that a diffraction grating based on an indium-tin oxide film is an amplitude-phase one. Firstly, the grating is characterized by differences in the transmittance of the step tB $=0.826$ and the trench $\mathrm{t} \kappa=0.910$ at the wavelength of $633 \mathrm{~nm}$ (fig. 3). Secondly, different phase changes correspond to the step and the trench, $\Delta \varphi \mathrm{B}=2.123 \mathrm{rad}$ and $\Delta \varphi \kappa=1.142 \mathrm{rad}$. These factors condition partial formation of odd orders, mainly the first ones that make up about $10 \%$ of the zero order.

Thus, a diffraction grating on the basis of ITO-films can be both a phase one if its thickness is a multiple of half-wavelength and the transmittance is maximum, and an amplitude one. The amplitude properties of the ITO-mask in this case are determined by its interference properties and optical absorption. It is an advantage of this kind of mask as compared to metal masks which are predominantly amplitude ones. On the other hand, optical parameters of an ITO-film may vary within fairly narrow limits after being produced, for example, with the help of annealing. This makes it possible to tune the diffraction grating parameters to the operating wavelength. Thus, additional 2-minute annealing of an ITO-film at a rate of $30{ }^{\circ} \mathrm{C} / \mathrm{min}$ up to the temperature of $250{ }^{\circ} \mathrm{C}$ resulted in some increase of the film transparency and considerable reduction of the film resistance from $50 \mathrm{ohm} / \mathrm{sq}$ до $26 \mathrm{ohm} / \mathrm{sq}$. This confirms the possibility 
of thermal change of its structure after production with the aim of tuning the parameters to the assigned wavelength.

\section{Conclusion}

A technology of formation of a conducting diffraction mask made of indium-tin oxide about $100 \mathrm{~nm}$ thick on the surface of a K-8 substrate is proposed in the paper. The analysis of the mask roughness showed good quality of its surface. The refractive index of an ITO-film $n=1.857 \pm 0.008$ and $k=0.000 \pm 0.009$ at the wavelength of 632.8 $\mathrm{nm}$ is determined by the method of ellipsometry. Spectral transmittance of the diffraction grating in the area of the step (of the mask) $t_{\mathrm{B}}=0.826$ and that of the trench tK $=0.910$ is measured and their difference caused by interference properties of the ITO-layer is noted. Intensity distribution in the focal plane of the ITO-grating is investigated and its amplitude-phase properties are shown. The measured dimensional and optical characteristics of the ITO diffraction mask are confirmed on the basis of the comparison of the design and measured directional diagrams. These data can be used for the design of transparent conducting masks of controlled diffraction gratings on substrates made of electro-optic materials. Variation of parameters of the amplitude-phase grating makes it possible to create laboratorial diffraction modulators of laser emission intensity.

Surface resistivity of ITO-electrodes is measured and the possibility of its thermal tuning almost two-fold, from $50 \mathrm{ohm} / \mathrm{sq}$ to $26 \mathrm{ohm} / \mathrm{sq}$ is demonstrated. This possibility is useful for matching complex resistance of a controlled diffraction grating with the output stage of the voltage source.

\section{Acknowledgements}

The work was supported of Russian scientific Foundation (grant 14-19-00114).

\section{References}

1. Karpeev SV, Khonina SN, Kharitonov SI. Study of the diffraction grating on the convex surface as a dispersive element. Computer Optics, 2015; 39(2): 211-217. [in Russian] DOI: 10.18287/0134-2452-2015-39-2-211-217.

2. Znamenskyi MYu, Lukashevich YaK, Skochilov AF, Fedulova NA. Transmissive threaded diffraction gratings for ultraviolet, visible and infrared regions of the spectrum. Journal of Optical Technology, 2014; 3: 51-54. [in Russian]

3. Karpeev SV, Pavelyev VS, Khonina SN, Kazanskiy NL, Gavrilov AV, Eropolov VA. Fibre sensors based on transverse mode selection. Journal of Modern Optics, 2007; 54(6): 833-844.

4. Garitchev VP, Golub MA, Karpeev SV, Krivoshlykov SG, Petrov NI, Sissakian IN, Soifer VA, Haubenreisser W, Jahn JU, Willsch R. Experimental investigation of mode coupling in a multi- 
mode graded-index fiber, caused by periodic microbends using computer-generated spatial filters. Optics Communication, 1985; 55(6): 403-405.

5. Berezny AE, Karpeev SV, Uspleniev GV. Computer-generated holographic optical elements produced by photolithography. Optics and Lasers in Engineering, 1991; 15(5): 331340.

6. Veiko VP, Korol'kov VP, Poleshchuk AG, Sametov AR, Shakhno EA, Yarchuk MV. Study of the spatial resolution of laser thermochemical technology for recording diffraction microstructures. Quantum Electron, 2011; 41(7): 631-636.

7. Alferov SV, Khonina SN, Karpeev SV. Study of polarization properties of fiber-optics probes with use of a binary phase plate. Journal of the Optical Society of America A., 2014; 31(4): 802-807.

8. Poleshchuk AG, Korol'kov VP, Sedukhin AG et al. Direct laser writing of gray-scale microimages with a large dynamic range in chromium films. Optoelectronics, Instrumentation and Data Processing (Avtometriya), 2015; 51(3): 87-93. [In Russian].

9. Volkov AV, Kazanskiy NL, Moiseev OYu, Paranin VD, Poletayev SD, Chistyakov IV. Specific features of the laser irradiation of thin molybdenum films. Technical Physics, 2016; 61(4): 579-583. DOI: 10.1134/S1063784216040241.

10. Kazanskiy NL, Moiseev OYu, Poletayev SD. Microprofile formation by thermal oxidation of molybdenum films. Technical Physics Letters, 2016; 42(2): 164-166. DOI: 10.1134/S1063785016020085.

11. Dyachenko PN, Karpeev SV, Fesik EV et al. The three-dimensional photonic crystals coated by gold nanoparticles. Optics Communications, 2011; 284(3): 885-888.

12. Song QW, Wang XM, Bussjager R. High-efficiency electro-optic grating switch with improved performance. Applied Optics, 1996; 45(35): 7031-7036.

13. Yang X, Aspelmeyer M, Wood LT, Miller JH. Diffraction from tunable periodic structures. II. Experimental observation of electric field-induced diffraction peaks. Applied Optics, 2002; 41(28): 5845-5850.

14. Ye Q, Dong Z, Qu R, Fang Z. Experimental investigation of optical beam deflection based on PLZT electro-optic ceramic. Optics Express, 2007; 14(25): 16933-44.

15. Ye Q, Qiao L, Cai H, Qu R. High-efficiency electrically tunable phase diffraction grating based on a transparent lead magnesium niobate-lead titanite electro-optic ceramic. Optics Letters, 2011; 36(13): 2453-2455.

16. Paranin VD. Methods to control parameters of a diffraction grating on the surface of lithium niobate electro-optical crystal. Technical Physics, 2014; 59(11): 1723-1727.

17. Kireev VYu. Introduction to technologies of microelectronics and nanotechnologies.Moscow: FGUP “CNIIHM” Publisher, 2008. 432 p. [in Russian]. 